\title{
Assembly of Janus complex with Low-cost and Salt Rejection for Solar- Thermal Water Evaporation
}

Dingding $\mathrm{Li}^{1}$, Qingxin Zhou ${ }^{1}$, Gang Wang ${ }^{1}$, Hang Zhao ${ }^{1}$, Shenghua $\mathrm{Ma}^{1,{ }^{*}}$, Kunyue Leng ${ }^{1, *}$, Yi Wang ${ }^{1, *}$, Jinbo Bai2,*

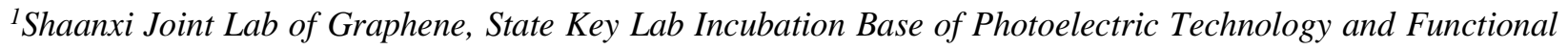
Materials, International Collaborative Center on Photoelectric Technology and Nano Functional Materials, Institute of Photonics \& Photon-Technology, Northwest University, Xi'an 710069, China

${ }^{2}$ Laboratoire Mécanique des Sols, Structures et Matériaux (MSSMat), CNRS UMR 8579, Ecole CentraleSupélec, Université Paris-Saclay, 8-10 rue Joliot-Curie, 91190 Gif-sur-Yvette, France

*Corresponding author

E-mail address: mashenghua@nwu.edu.cn (S.M.) lengky@nwu.edu.cn (K.Y.) yi.wang@nwu.edu.cn (Y.W.) and jinbo.bai@centralesupelec.fr (J.B.). 


\begin{abstract}
Interfacial solar-thermal water evaporation (STWE) shows considerable potential in terms of harvesting fresh water from the ocean, thereinto the design of efficient floatable material with low-cost and salt deposition resistant always one of most concerned issue. In this work, we prepared an economical Janus complex for STWE by assembling commercial carbon nanotubes and porous cotton fiber paper, functionalized by silanization and combination of polyelectrolyte. Because of the synergy of efficient photothermal conversion, localized hot layer and fast transmission of water and vapor, the Janus complex displayed superior evaporation rate of 3.443 $\mathrm{kg} \cdot \mathrm{m}^{-2} \cdot \mathrm{h}^{-1}$ under 5 suns $\left(5 \mathrm{~kW} \mathrm{~m} \mathrm{~m}^{-2}\right)$ in saltwater. Moreover, almost no salt was detected in the used Janus complex, the salt deposition was rejected at the source due to the strong ionic strength of polyelectrolyte. These results indicated that the Janus complex has potential applications for water purification.
\end{abstract}

Key words: carbon nanotube; Janus complex; salt rejection; polyelectrolyte; solar-thermal water evaporation 


\section{Introduction}

How to alleviate the shortages of drinking water has long been a global problem as the rapid prosperity of the world, especially in underdeveloped regions [1,2]. Thereinto, the most concern may purify water with sustainable technology [3]. As this concern, collecting drinking water from ocean through water evaporation derived by solar harvesting has aroused great attention, due to the abundant reserves and economical sustainable of solar energy [4,5]. Light harvesting is commonly a direct process that absorbing solar radiation in a heat transfer fluid, but to promote water collection, an indirect process involves the absorption of incident photons and heat transfer from light absorbing surface to surrounding media is usually practiced over solar harvesting materials. To achieve this indirect process, efficient solar harvesting materials need to be elaborated. Currently, various materials have been developed to face the challenge [6], especially the materials with "black photothermal" nature [7], such as single or multi-layer carbon-based materials [8-10], individual or decorated plasmonic structure [11-13], diverse semiconductor sheets [14-16], monolithic polymer foam [17] and hydrogel/ aerogel [18].

According to current researches we reckoned the applicable evaporator should have not only the characteristics of high photothermal conversion efficiency [19] and restriction of heat dissipation [20], but also the efficient transmission between liquid water and heat source [13] and short residence time of water steam [8]. Janus complex was one of the most promising thin layer materials to face all the demands. This material was first reported by Cho and Lee in 1985 as a unique structure with two harmonious sides and different functions [21-23], and has been applied in various fields, such as oil separation [24], $\mathrm{CO}_{2}$ capture [25], defogging [26], solar water evaporation [27-30], et al. In solar-thermal water evaporation (STWE) applications, except 
photothermal function, Janus evaporator also manifests itself as a combination of hydrophobic and hydrophilic sides. The hydrophobic side is usually consisted of modified light-absorbing material such as carbon [31-34] and metallic oxide/ sulfide [35, 36], while the hydrophilic side is made of superwetting materials with water pumping ability, thermal insulation, and robustness [37-40]. The hydrophilic surface could adsorb water from the matrix and transfer it to the light absorbing surface, and the hydrophobic light absorbing surface conduct heat to adsorbed water and accelerate water vapor to leave the evaporator. By the collaboration of the "two faces", STWE performance of Janus complex could be boosted significantly [27]. Moreover, the preparation cost of Janus evaporators requires careful calculation to face the potential applications [41-44].

Despite various Janus complexes have been designed and used in the STWE process, there was still a challenge when solar desalination was involved due to the abundant inorganic metal salt in seawater. The drawback is that the localized-heating and rapid interfacial evaporation will cause the salt deposition on the surface and in the interspace of the hydrophobic layer, results in the weakening of STWE performance as time passes. While the frequent regeneration requires additional costs and more robust structure. Many efforts have been made to solve this problem, such as enhance salt dilution by polystyrene foam layers based on a diffusion and advection mechanism [45], isolate hydrophobic surfaces from salt-water [29,46], and boost salt excretion properties by low tortuosity pore structure material [27]. But suppressing salt deposition from the source was still be considered the best solution. Recently, R. Chen and co-workers brought the idea that establishing solar desalination material with polyelectrolyte to light [47], as reported ions were rejected at the solution/foam interface when a specific hydrogel foam was used for 
desalination due to the strong ionic strength of polyelectrolyte. This concept may solve saltdeposition fundamentally.

Herein, in order to make full use of the advantages of light harvesting materials and Janus structure, we reported a new device strategy of Janus complex by simply assembling of several facile commercial materials: carbon nanotube, porous cotton-fiber-paper (PCFP) and polyelectrolyte (PDDA). The cost was estimated to be $5.3 \$ / \mathrm{m}^{2}$ [44], which was comparatively low. The synergy of multifunctional surfaces makes this Janus complex displays good STWE performance and satisfactory reusability of 20 cycles. STWE test in model seawater revealed superior ion removal rates of over 99.9\%, moreover the Janus complex displayed excellent salt tolerance due to the strong ionic strength of polyelectrolyte, almost no salt was detected in the used evaporator. This amphiphilic structure provides a new concept to establish low-cost thin film material for efficient interfacial water purification with solar energy.

\section{Experimental}

\subsection{Materials}

N-hexane and $\mathrm{HNO}_{3}$ were provided by Xilong Chemicals (China). Octadecyltrimethoxysilane (ODTS) and poly-diallyldimethylammonium chloride (PDDA) were purchased from SigmaAldrich (China). Ultrapure water (18.2 $\mathrm{M} \Omega \cdot \mathrm{cm}$, Millipore Co.,USA) was used throughout. CNTs were obtained from Chengdu Organic Chemicals (China). Porous cotton fiber paper (PCPF) (diameter: 90mm; thickness: 0.168mm) was provided by Hangzhou Special Paper Co., Ltd. (Hangzhou, China).

\subsection{Preparation of Janus complex}


In order to silanize the multi-walled carbon nanotubes, the CNTs was pretreated by the oxidation of $\mathrm{HNO}_{3}$. Typically, $0.4 \mathrm{~g}$ of CNTs were mixed with $100 \mathrm{~mL}$ of a $3.0 \mathrm{M} \mathrm{HNO}_{3}$ solution and sonicated at $60{ }^{\circ} \mathrm{C}$ for 15 minutes. Then, the mixed liquid was filtered and repeatedly washed to cleanness, and then dried at $150{ }^{\circ} \mathrm{C}$ for 4 hours in a forced air oven [48], the obtained sample was labelled as OCNTs. In a typical assembly process, OCNTs was first modified by ODTS, 9 mg of OCNTs was added to $10 \mathrm{~mL}$ of hexane containing $100 \mu \mathrm{L}$ of ODTS, and sonicated for 15 $\min$ [49]. Then the mixture was mixed with a volume of PDDA aqueous solution (20 $\left.\mu \mathrm{L} \mathrm{mL}^{-1}\right)$ and vortex for 1 minute. The hexane is removed by standing separation, and then ethanol is added. After $12 \mathrm{~h}$ at room temperature $\left(25^{\circ} \mathrm{C}\right)$, The suspension was evenly dropped on the PCFP and dryed, the obtained Janus complex was labeled as t-OCNTs/PCFP JC. As reference, CNTs was assembled with PCFP at the same condition but without the functionalization of ODTS and PDDA, this sample was labeled as CNTs/PCFP complex.

\subsection{Characterizations}

Transmission electron microscopy (TEM) images were obtained using a Tecnai $\mathrm{G}^{2}$ F20 S-TWIN optics instrument. Scanning electron microscopy (SEM) images were obtained using a JEOL JSM-6390A instrument. X-ray diffraction (XRD) analysis was performed using a Rigaku Smart Lab diffractometer equipped with parallel beam optics; $\mathrm{Cu}-\mathrm{K} \alpha$ radiation $(40 \mathrm{kV}, 100 \mathrm{~mA})$ was incident at a grazing angle of $0.3^{\circ}$. The absorptions across $200-2000 \mathrm{~nm}$ of all complexes were measured by using Cary 5000 Scan UV-Vis-NIR spectrophotometer (Varian, USA) equipped with an integrating sphere. X-ray photoelectron spectroscopy (XPS) spectra of the samples were collected on PHI-5400 electron spectrometer. Thermal gravimetric analysis (TGA, SDT Q600) was measured in nitrogen atmosphere at a heating rate of $10{ }^{\circ} \mathrm{C} / \mathrm{min}$ from room temperature to $1000^{\circ} \mathrm{C}$. Raman spectroscopy was detected on a Nicolet Almega dispersive Raman spectrometer 
with laser excitation. The contact angle images were taken on a contact angle analyzer (DSA 100, KRUSS incorporation in German). The Fourier transform infrared (FT-IR) spectra were obtained on Bruker Optics Tensor 27 spectrometer. The $\mathrm{N}_{2}$ adsorption-desorption analysis was taken on Tristar II3020 apparatus.

\subsection{Solar Steam Generation Measurements}

Solar steam generation experiments were performed in a custom optical measurement system, including a multi-function solar simulator (PL-X300DF, Beijing Precise Technology Co., Ltd., China) with an AM 1.5 filter. The evaporation rate is measured by a high precision balance (FA 2004, accuracy $0.1 \mathrm{mg}$ ). Solar illuminance was monitored using a Thorlabs PM100D power meter from 1 to $5 \mathrm{~kW} \mathrm{~m}$. FLIR (T620, USA) camera used to measure the maximum temperature of the Janus and CNTs/PCFP complex under various solar concentrations. The seawater desalination experiment was tested by a homemade evaporative water collection system. Simulating natural seawater by salt solution, the initial concentrations of $\mathrm{NaCl}, \mathrm{MgCl}_{2}, \mathrm{KCl}$ and $\mathrm{CaCl}_{2}$ in the seawater were $7000,1425,1268$, and $295 \mathrm{mg} \mathrm{L}^{-1}$, respectively. The concentration of the $\mathrm{NaCl}$ solution used for desalination is $7000 \mathrm{mg} \mathrm{L}^{-1}$. The salinities of natural seawater and collected water were characterized by inductively coupled plasma-optical emission spectroscopy (ICP-OES).

\section{Results and discussion}




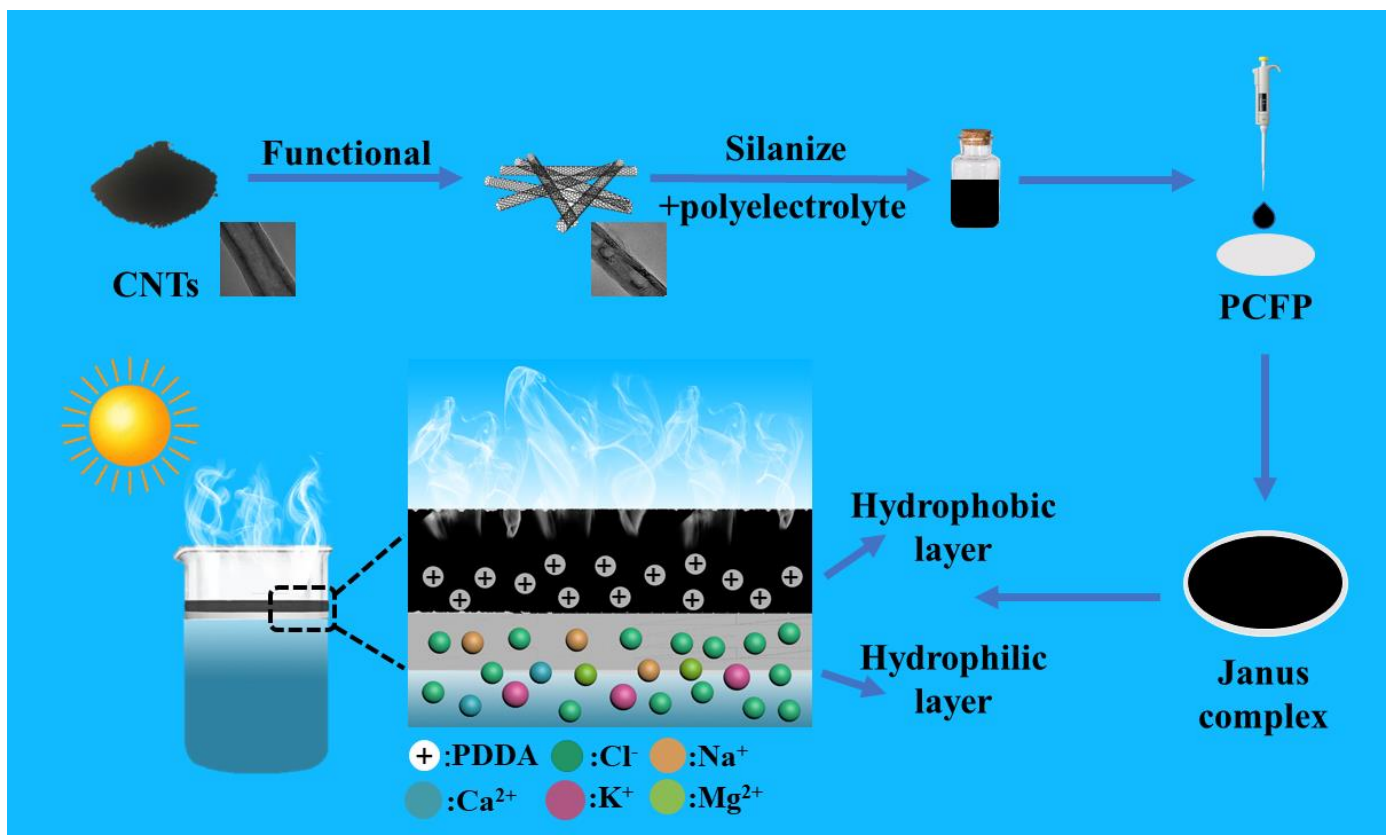

Figure 1 Assemble process, structure diagram and solar-thermal water evaporation process of Janus complex.

As shown in Fig. 1, the Janus complex used in this work was consisted of two layers and was floating on the water during STWE process. The hydrophilic substratum is PCFP that contacts with water and serves as water transmission medium (by capillarity) and heat shield (due to the poor thermal conductivity). The hydrophobic upper layer exposed in the air that acted as photothermal converter and calorifier is combined with carbon nanotubes, ODTS and PDDA. PDDA is a type of polyelectrolyte, as Chen's report the addition of polyelectrolyte in STWE material could enhance the salt deposition resistant [47]. According to the market prices of the main raw materials $\left(0.14 \$ / \mathrm{m}^{2}\right.$ for PCPF and $0.08 \$ / \mathrm{g}$ for CNTs), the cost of Janus complex was roughly estimated to $5.3 \$ / \mathrm{m}^{2}$. 

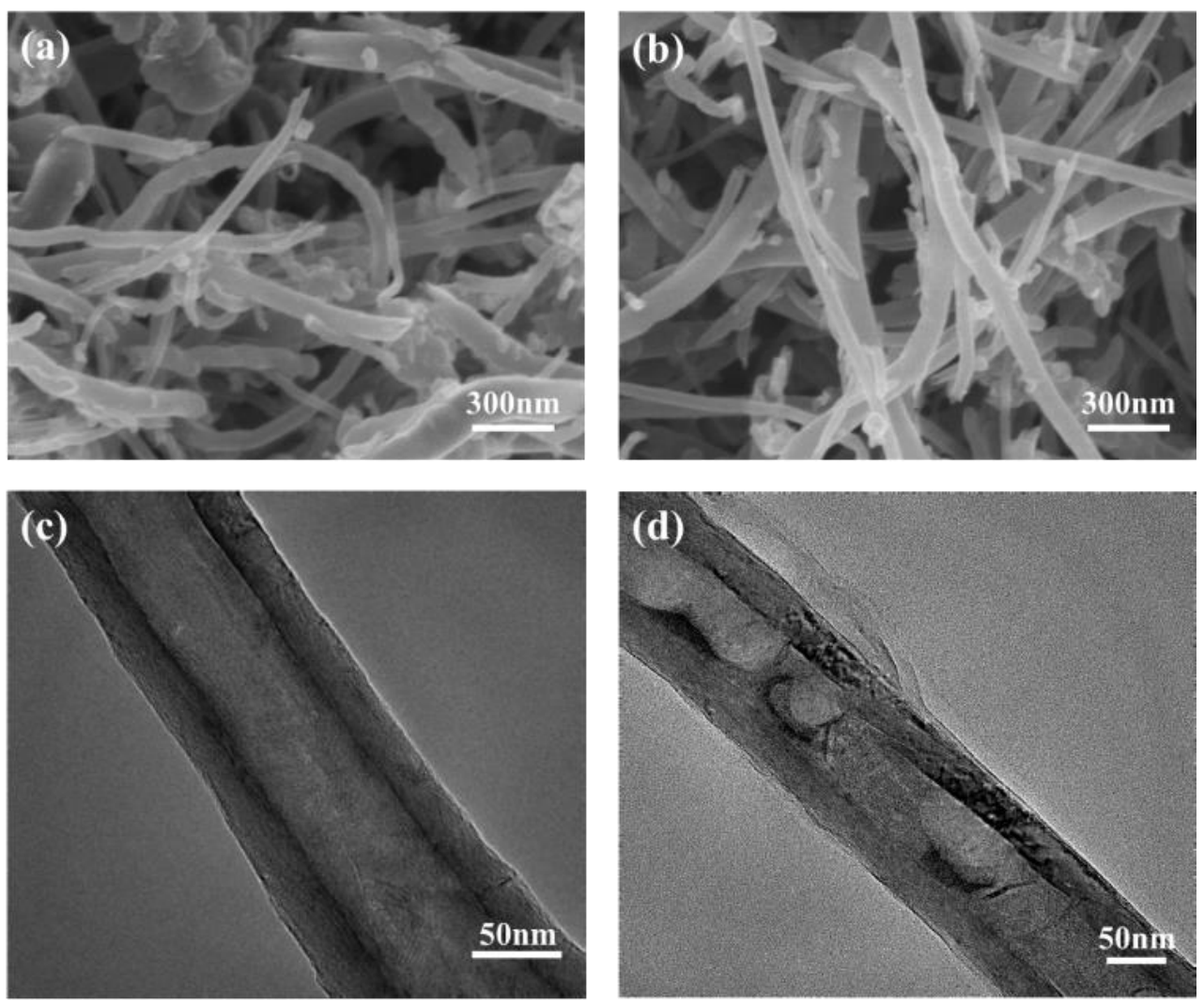

Figure 2 SEM images of (a) CNTs and (b) OCNTs. TEM images of (c) CNTs and (d) OCNTs.

In order to improve the hydrophobicity by silanization, CNTs was first pretreated with $\mathrm{HNO}_{3}$. SEM and TEM images of various carbon nanotubes were shown in Fig. 2. It can be seen from SEM images that after pretreatment OCNTs displayed semblable length and diameter with CNTs (Fig. 2a, b), but TEM images showed varying degrees of etching on the wall of OCNTs (Fig. 2c, d). The results of the TGA test and Nitrogen adsorption-desorption test also confirmed that $\mathrm{HNO}_{3}$ pretreatment had no distinct influence on the thermostability and porous structure (Figure S1, S2, Supporting Information). These results indicated that $\mathrm{HNO}_{3}$ pretreatment could result in the formation of defects on CNTs but with negligible influence on the morphology. 

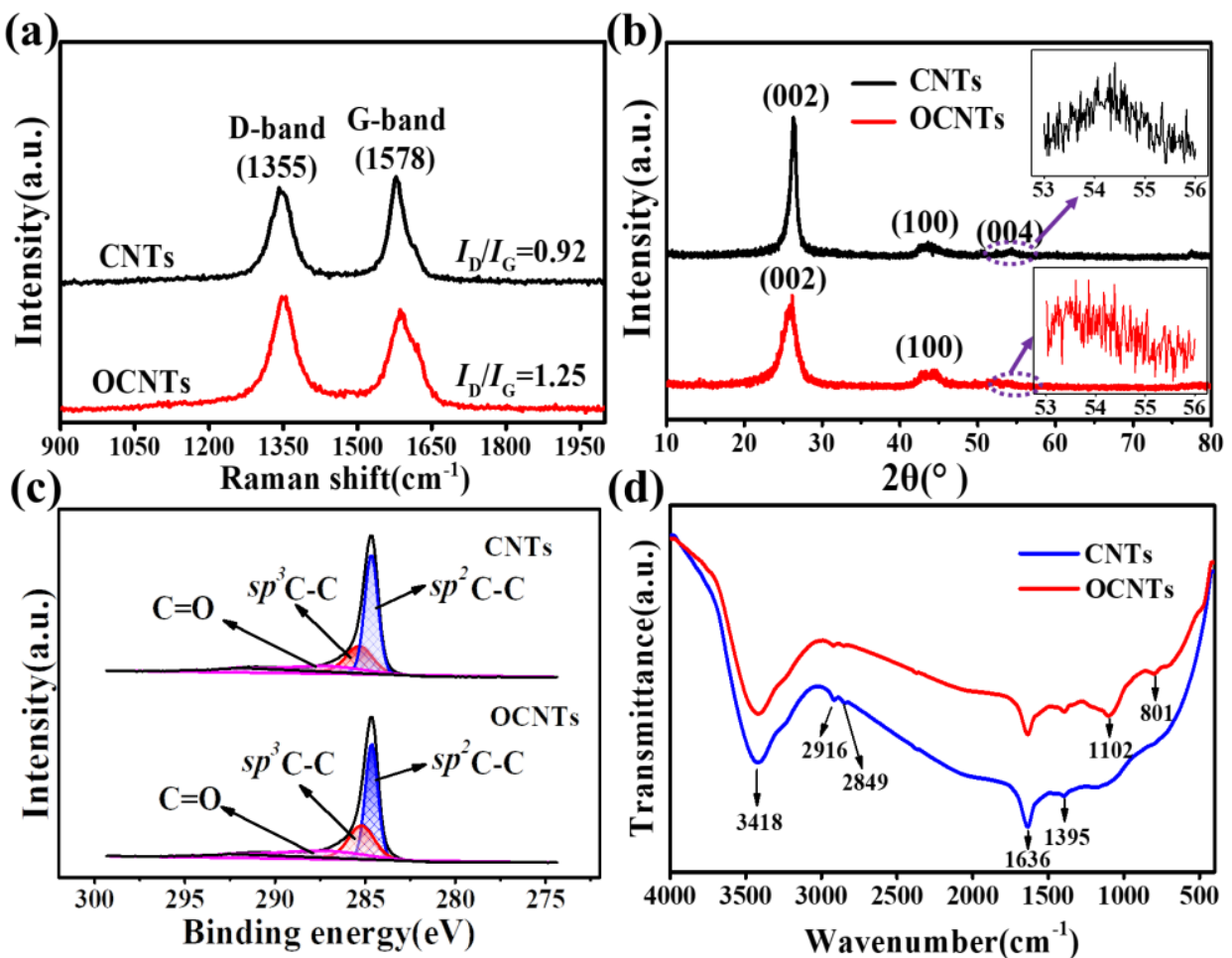

Figure 3 (a) Raman spectra of the CNTs and OCNTs. (b) XRD pattern of the CNTs and OCNTs. (c) The C1s XPS spectra of CNTs and OCNTs. (d) FTIR spectra of the CNTs and OCNTs.

To further demonstrate the formation of defects, the structural characteristics of various samples were investigated. Raman spectroscopies were obtained and shown in Fig. 3a. It is well known that the absorption around 1,355 and $1,578 \mathrm{~cm}^{-1}$ were due to the $\mathrm{D}$ and $\mathrm{G}$ bands of carbon nanotube, which related to the defect of the $\mathrm{C}$ atomic lattice and the in-plane stretching vibration of the $\mathrm{C}$ atom $\mathrm{sp}^{2}$ hybridization, respectively. It can be seen that the ratio of peak intensities $\left(\mathrm{I}_{\mathrm{D}} / \mathrm{I}_{\mathrm{G}}\right)$ of OCNTs increased from 0.92 to 1.25 compared to CNTs, revealed that more defects were formed after $\mathrm{HNO}_{3}$ pretreatment. To further verify the effect of $\mathrm{HNO}_{3}$ pretreatment, Fig. 3b shows the XRD patterns of various samples, although the diffraction of (004) face of OCNTs was disappeared substantially, it still maintained the diffraction of (002) and (100) face same as the pattern of CNTs. It was confirmed again that $\mathrm{HNO}_{3}$ pretreatment would reduce the graphite 
carbon without excessive destructing the structure framework. The results of Raman spectroscopies and XRD consistent well with the TEM and SEM images.

The results of C 1s XPS were shown in Fig. 3c, as seen both samples exhibited three peaks centred at binding energy of $284.64 \mathrm{eV}, 285.18 \mathrm{eV}$ and $287.23 \mathrm{eV}$ after deconvolution, related to bonds of $\mathrm{sp}^{2} \mathrm{C}-\mathrm{C}, \mathrm{sp}^{3} \mathrm{C}-\mathrm{C}$ and $\mathrm{C}=\mathrm{O}$ respectively. The result of OCNTs displayed higher $\mathrm{C}=\mathrm{O}$ content but less $\mathrm{sp}^{2} \mathrm{C}-\mathrm{C}$ content, indicated that there were more oxygen-containing groups in OCNTs. The existence of oxygen-containing groups was further confirmed by FT-IR spectrum which was shown in Fig. 3d. It can be seen that OCNTs displayed obvious absorption around 1395 and $1102 \mathrm{~cm}^{-1}$, which was assigned to $-\mathrm{OH}$ bending deformation present in $\mathrm{COOH}$ and $\mathrm{C}-\mathrm{O}$ stretch in alcohols (the absorption around $801 \mathrm{~cm}^{-1}$ was assigned to aromatic C-H outof-plane bending vibrations) [50]. As reported, these oxygen-containing groups were conducive to the post-modification with ODTS [51].

After $\mathrm{HNO}_{3}$ pretreatment, the OCNTs was used as primary raw material to assemble Janus complex. Typically, the modified CNTs form a polymer with the negatively charged ODTS, and then the positively charged PDDA is combined with the negatively charged polymer, drop the mixed liquid on the hydrophilic PCFP [34], after aged and dried the t-OCNTs/PCFP Janus complex (JC) was obtained. Form the results of FT-IR in Figure S4 it can be seen, t-OCNTs displayed two absorptions around 901 and $720 \mathrm{~cm}^{-1}$ that assigned to O-Si-O and Si-C bond, revealed the successful combination of ODTS in t-OCNTs/PCFP JC (the result of EDS mapping also confirm the existence of Si, Figure S5, Supporting Information). But there was no such absorption in the FT-IR spectrum of reference samples due to the lacking of requisite functional 
groups. The absorption around $1466 \mathrm{~cm}^{-1}$ was assigned to characteristic $\mathrm{C}=\mathrm{C}$ band of PDDA, confirmed the combination of PDDA.

(a)

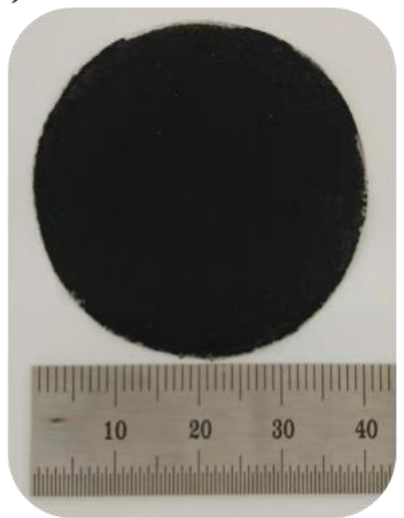

(c)

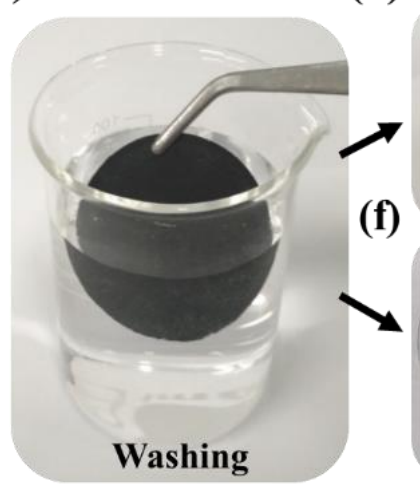

(b)

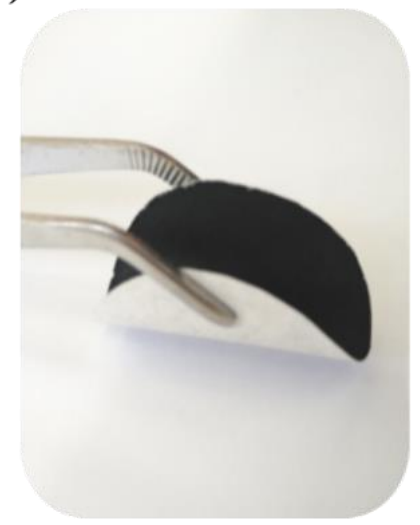

(e)

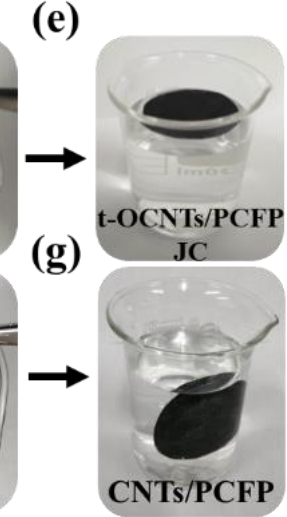

(h)

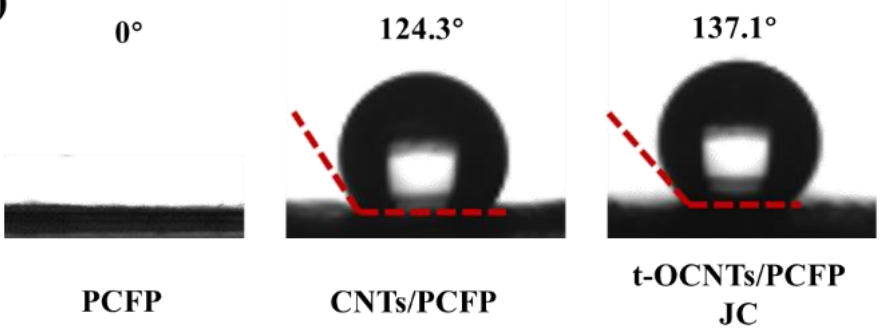

Figure 4 Digital photograph of t-OCNTs/PCFP JC. (a) original, (b) after be curved, (c) before and (d, e) after be infiltrated in water. (f, g) Digital photograph of CNTs/PCFP complex after infiltrated in water. (h) Water contact angle of PCFP, CNTs/PCFP complex and t-OCNTs/PCFP JC.

As a flexible material, t-OCNTs/PCFP JC could be cut into different shapes and sizes, and be curved over 150 degree, based on the various applications (Fig. 4a, b). It is worth noting that 
the t-OCNTs/PCFP JC also displayed good native floatability, it could still float on the surface after infiltrated in water (Fig. 4c-e), while the reference samples CNTs/PCFP complex sank to the bottom after same treatment (Fig. 4f, g). This was benefit from the better hydrophobicity caused by the modification of ODTS, as shown in Fig. 4h and Figure S6, t-OCNTs/PCFP JC maintained a contact angle of $139.6^{\circ}$ even after 5 s contacted with water droplets, $22.6^{\circ}$ greater than that of CNTs/PCFP complex $\left(117.0^{\circ}\right)$. While for PCFP, the water droplets were absorbed in just $0.14 \mathrm{~s}$, such superior capillarity may make PCFP an excellent pump for water transmission. The results of water contact angle also revealed that the two layers of t-OCNTs/PCFP JC have completely opposite properties in terms of water affinity, which is just accord with the concept of Janus.

(a)

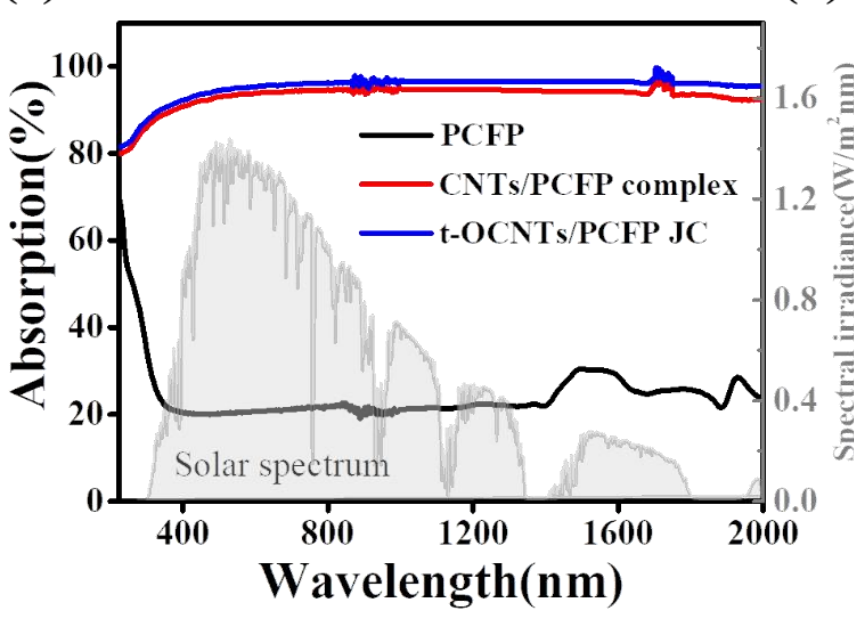

(b)

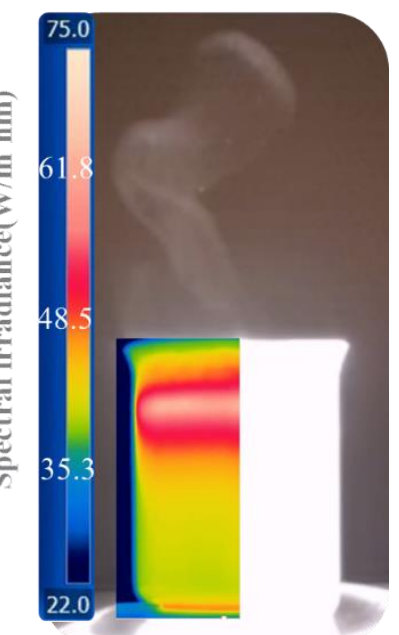

Figure 5 (a) UV-vis-NIR absorption spectrum of PCFP, CNTs/PCFP complex and t-OCNTs/PCFP JC. (b) Images of water evaporation.

To our surprise, although introduced light-insensitive groups, the modification of ODTS and PDDA seemed to have no disadvantage to solar absorption capacity. As shown in Fig. 5a, UV-vis-NIR absorption spectrum of t-OCNTs/PCFP JC displayed 95\% average absorption between the wavelength of $230-2000 \mathrm{~nm}$ (weighted by AM1.5G solar spectrum), even a little 
higher than that of CNTs/PCFP complex (93\%). The above results inspired us that the synergy of high average absorption in a wide range and superior hydrophobicity may make this Janus complex an excellent thin film for interfacial STWE. It can be seen from Fig. 5b, solar was converted to thermal energy by carbon nanotubes and formed localized hot layer around the film due to the low thermal conductivity of PCFP, then water was pumped to the hot zone, evaporated and excreted from the hydrophobic surface quickly (the generation for steam was quite evident in the digital photograph).

Solar-thermal Water Evaporation Test 

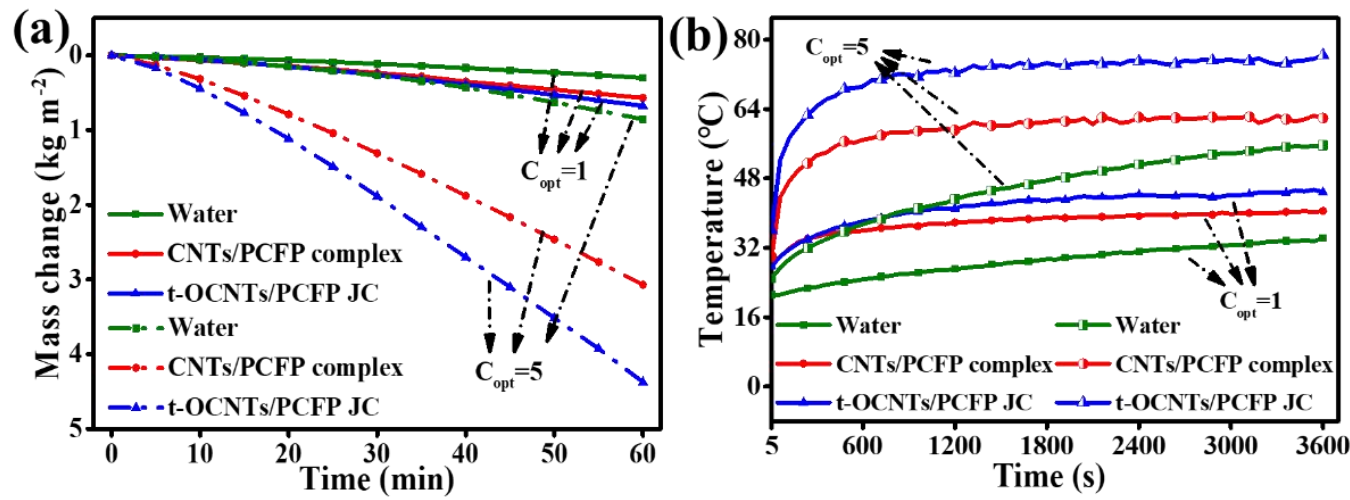

(c)

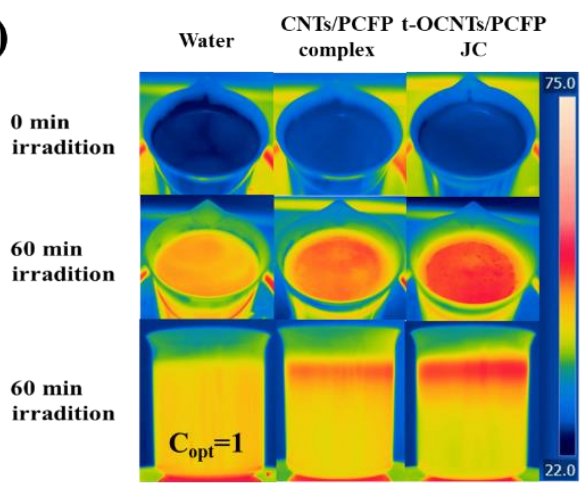

(d)

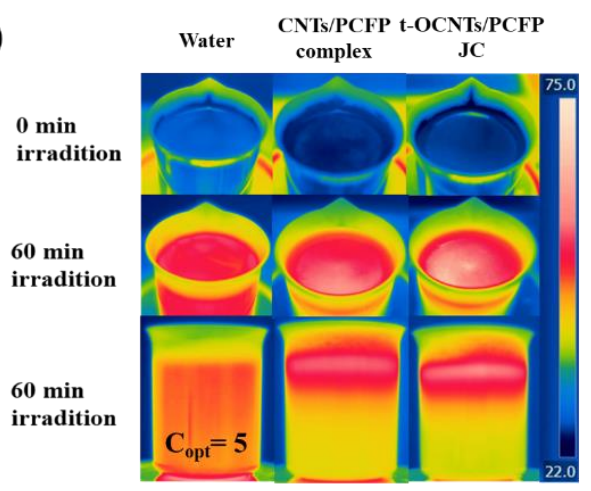

(e)

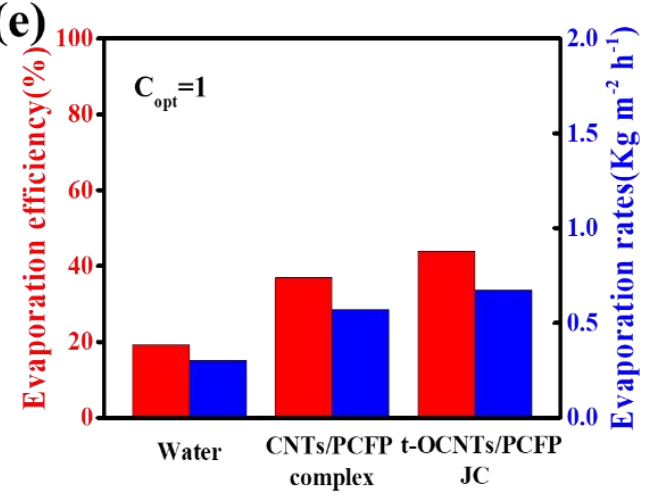

(f)

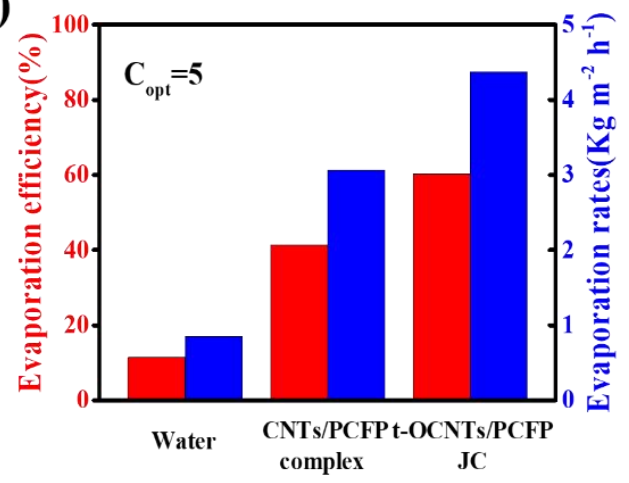

Figure 6 (a) Mass change of water over time of various samples under 1 and $5 \mathrm{~kW} \mathrm{~m}^{-2}$ solar irradiation, (b) Surface temperature change over time of various samples. (c, d) IR images of various samples under different solar irradiation. (e, f) Evaporation rate and evaporation efficiency of various samples.

To demonstrate the potential superiority of t-OCNTs/PCFP JC, STWE performance was evaluated by evaporating water with optical concentrations $\left(\mathrm{C}_{\mathrm{opt}}\right)$ of 1 and 5 (which correspond to energy density of 1 and $5 \mathrm{~kW} \mathrm{~m}^{-2}$ ). Fig. 6 a shows the mass change of water with time over various complexes and conditions. As seen, when $\mathrm{C}_{\mathrm{opt}}$ was 1 the mass change over $\mathrm{t}$ - 
OCNTs/PCFP JC was $0.674 \mathrm{~kg} \mathrm{~m}^{-2}$ after exposure for $60 \mathrm{~min}$, about 1.2 times that of over CNTs/PCFP complex $\left(0.569 \mathrm{~kg} \mathrm{~m}^{-2}\right)$, and 2.2 times that of for pure water $\left(0.301 \mathrm{~kg} \mathrm{~m}^{-2}\right)$. As the $\mathrm{C}_{\text {opt }}$ increased to 5 , mass change over t-OCNTs/PCFP JC increased to $4.374 \mathrm{~kg} \mathrm{~m}^{-2}$ (enhanced by $549 \%$ ), while that over CNTs/PCFP complex was just $3.069 \mathrm{~kg} \mathrm{~m}^{-2}$ (enhanced by $439 \%$ ). To further investigate the evaporation process, the temperature distribution of the STWE system was obtained by infrared (IR) camera (Fig. 6c, d). As seen, no matter under 1 or $5 \mathrm{C}_{\mathrm{opt}}$, pure water system displayed no temperature gradient from top to bottom. But for t-OCNTs/PCFP JC and CNTs/PCFP complex, there was visible localizing hot layer. This hot layer concentrates most of solar-heat at the surface between water and air, so the generation of steam was enhanced. Moreover, as seen in Fig. $6 \mathrm{~b}$ the interfacial water temperature of t-OCNTs/PCFP JC rapid increased to $44.8^{\circ} \mathrm{C}\left(\mathrm{C}_{\mathrm{opt}}=1\right)$ or $74.6^{\circ} \mathrm{C}\left(\mathrm{C}_{\mathrm{opt}}=5\right)$ in the initial $600 \mathrm{~s}$ and maintained until the end of STWE process, which was apparently higher than that of CNTs/PCFP complex and the water under the interface (Figure S10-12, Supporting Information). The higher interfacial temperature and faster heating rate revealed the superiority of t-OCNTs/PCFP JC in STWE, which was further demonstrated by the result of evaporation rate and evaporation efficiency as shown in Fig. $6 e, f$. Thermal efficiency is defined as

$$
\eta=\frac{\dot{\mathrm{m}} h_{L V}}{q_{i} C_{o p t}}
$$

where $\dot{m}$ is the mass change, $\mathrm{h}_{L V}$ denotes the total enthalpy of liquid-steam phase change (2575 $\mathrm{kJ} \mathrm{kg}^{-1}$ ) which was obtained by adding sensible heat $\left(315 \mathrm{~kJ} \mathrm{~kg}^{-1}\right.$, from 25 to $100{ }^{\circ} \mathrm{C}$ with specific heat of $\left.4.2 \mathrm{~kJ} \mathrm{~kg}^{-1}\right)$ and the latent heat of vaporization of water $\left(\sim 2260 \mathrm{~kJ} \mathrm{~kg}^{-1}\right)$, and $\mathrm{q}_{i} \mathrm{C}_{\text {opt }}$ is the total light energy supplied to the film by the light source [52]. As seen, the evaporation rate and evaporation efficiency of t-OCNTs/PCFP JC were about $20 \%$ higher than 
that of CNTs/PCFP complex when $\mathrm{C}_{\mathrm{opt}}=5$. The influence of the content of carbon nanotubes and PDDA in t-OCNTs/PCFP JC were also studied, the results of STWE test showed no apparent difference (Figure S13 and S14, Supporting Information). In order to evaluate the stability of the t-OCNTs/PCFP JC, the STWE experiment was repeated several times under the same conditions. It is worth noting that t-OCNTs/PCFP JC displayed good stability that could be reused at least 20 cycles (Figure S15, Supporting Information).
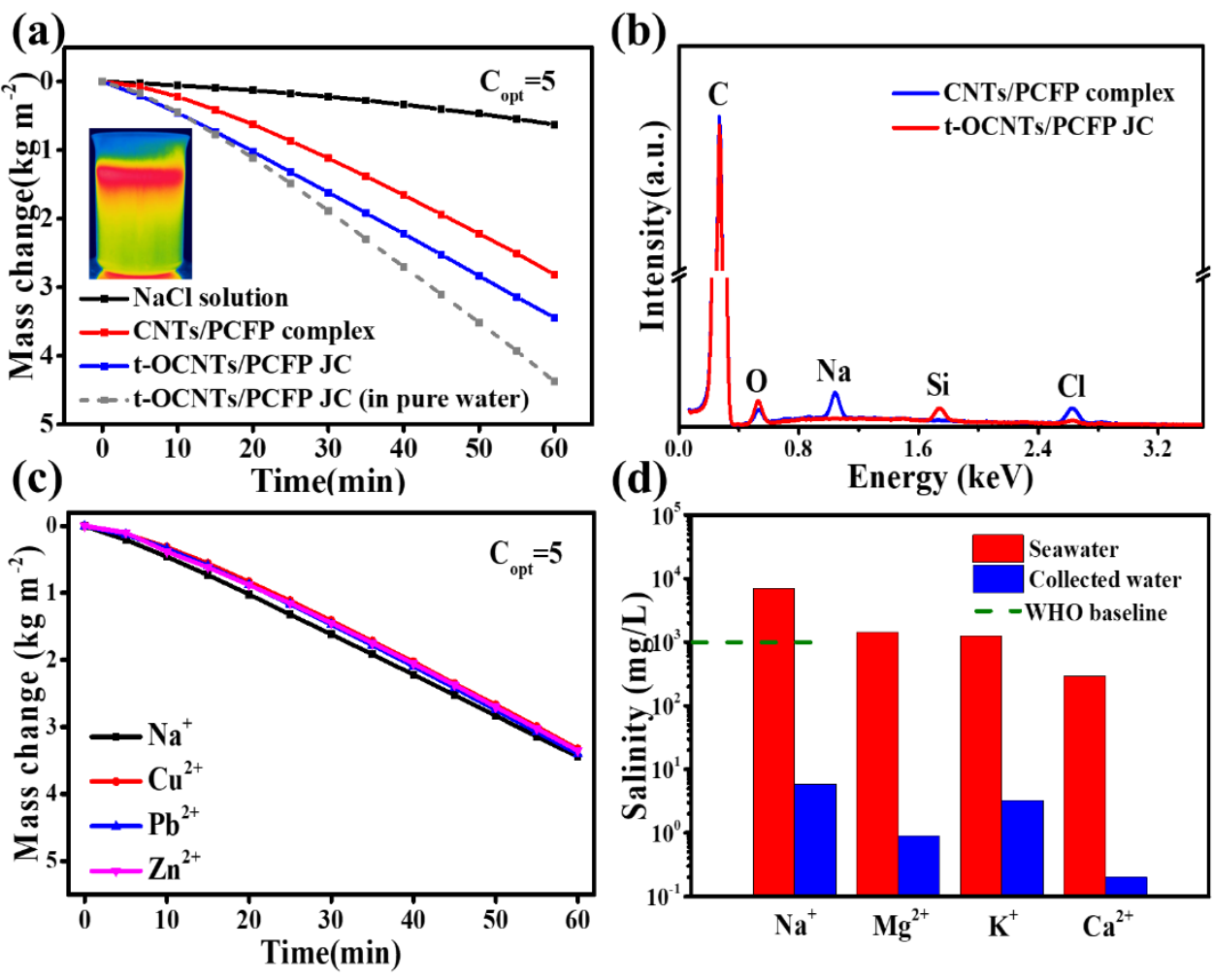

Figure 7 (a) Mass change over time of various samples in $\mathrm{NaCl}$ solution under $5 \mathrm{~kW} \mathrm{~m} \mathrm{~m}^{-2}$ solar irradiation. (b) EDS spectra of CNTs/PCFP complex and t-OCNTs/PCFP JC after used. (c) Mass change over time of t-OCNTs/PCFP JC in various salt water under $5 \mathrm{~kW} \mathrm{~m}^{-2}$ solar irradiation. (d) The typical cations in seawater and collected water after desalinization by $\mathrm{t}-\mathrm{OCNTs} / \mathrm{PCFP} \mathrm{JC}$.

To our best knowledge, seawater desalination is one of the main applications of STWE process, so the STWE performance of t-OCNTs/PCFP JC was first tested in $\mathrm{NaCl}$ solution at $\mathrm{C}_{\mathrm{opt}}$ $=5$ for 60 min and shown in Fig. 7a. As the same in pure water, after irradiated t-OCNTs/PCFP 
JC exhibited a localizing hot layer around surface and displayed a better mass change $(3.443 \mathrm{~kg}$ $\left.\mathrm{m}^{-2}\right)$ than CNTS/PCFP complex $\left(2.810 \mathrm{~kg} \mathrm{~m}^{-2}\right)$ and $\mathrm{NaCl}$ solution only $\left(0.626 \mathrm{~kg} \mathrm{~m}^{-2}\right)$.

As mentioned before, the main challenge to design STWE material for desalination is not how to generate water steam but how to suppress salt deposition which would reduce the lifetime of materials. To meet such challenge, polyelectrolyte PDDA was merged into the tOCNTs/PCFP JC successfully at the synthesis stage, and we hope the strong ionic strength of PDDA could suppress salt deposition at the source. To demonstrate the feasibility of this design, the t-OCNTs/PCFP JC and contrast samples were collected after STWE test in NaCl solution and analyzed by EDS. As shown in Fig. 7b, the EDS results of used t-OCNTs/PCFP JC almost displayed no signal of $\mathrm{Cl}$ and $\mathrm{Na}$, but for reference samples, such as CNT/PCFP complex (without ODTS and PDDA), OCNTs/ODTS/PCFP complex (without PDDA) and PCFP (hydrophilic layer), the signal of $\mathrm{Cl}$ and $\mathrm{Na}$ were all quite obvious (Fig. 7b, Figure S16-19, Supporting Information). These results clarified the pivotal role of PDDA in salt suppression process. Theoretically, evaporation process was accomplished in the hydrophobic layer, according to Chen's report, the ionic shield of polyelectrolyte like PDDA could hold the salt back in the hydrophilic layer, which will redissolve and go back to the water matrix (Fig. 1).

The STWE performance of t-OCNTs/PCFP JC was also tested with other salt solution $\left(\mathrm{Cu}^{2+}\right.$, $\mathrm{Pb}^{2+}, \mathrm{Zn}^{2+}$, et al.) and model seawater, as shown in Fig. 7c-d and Figure S21, excellent ion removal rates over $99.9 \%$ were observed over all salt solution, the salt contents in condensate water were reduced at least three orders of magnitude $(5.76,0.89,3.24,0.2,0.14,0.11$ and 0.19 mg L $\mathrm{L}^{-1}$ for $\mathrm{Na}^{+}, \mathrm{Mg}^{2+}, \mathrm{K}^{+}, \mathrm{Ca}^{2+}, \mathrm{Cu}^{2+}, \mathrm{Pb}^{2+}$ and $\mathrm{Zn}^{2+}$, respectively), which was in line with the salinity levels set by the World Health Organization (WHO). Taking into account the comparable desalination capacity with other attractive Janus structures (Table S2, Supporting Information) 
[27-30,53,54], and characteristics of metal-free and salt resistance, t-OCNTs/PCFP JC makes itself potentially applicable. But the photothermal efficiency of the device is limited to a certain extent due to the absence of metal additive, which may be solved by developing efficient and low-cost metal-free hydrophobic layer in future research.

\section{Conclusion}

In summary, we reported a low-cost Janus complex t-OCNTs/PCFP JC for highly efficient interfacial solar water evaporation. With the aim to boost the hydrophobicity of t-OCNTs/PCFP JC, CNTs was treated by sequential $\mathrm{HNO}_{3}$ pretreatment and ODTS modification, then assembled with PDDA and PCFP. In typical STWE process, the requisite localizing hot layer which is conducive to the generation of steam was formed due to the poor thermal conductivity of PCFP. Simultaneously, steam was fast excreted benefit by the superior hydrophobicity. As a result, $\mathrm{t}$ OCNTs/PCFP displayed solar water evaporation performance of $3.443 \mathrm{~kg} \cdot \mathrm{m}^{-2} \cdot \mathrm{h}^{-1}$ and excellent ion removal rates over $99.9 \%$ in model seawater. Moreover, salt deposition was rejected during the solar desalination process due to the strong ionic strength of PDDA. Such a result suggesting that the t-OCNTs/PCFP has the potential for application in water purification.

\section{Acknowledgements}

This work was supported by National Natural Science Foundation of China (Grant No. 51873134), and Natural Science Foundation of Shaanxi Province (No.2018JQ5058), Industrial Innovation China of Key Research, Development Project of Shaanxi Province (No. 2019ZDLGY16-09), and Scientific Research Fund of Shaanxi Provincial Education Department (No.19JK0861 and 20JK0945) and China Postdoctoral Science Foundation (No. 2020M673461). 


\section{Compliance with ethical standards}

Conflicts of interest The authors declare no conflict of interest.

Electronic Supplementary Material: The online version of this article (http://dx.doi.org/10.1007/s12274-***_******) contains supplementary material, which is available to authorized users.

\section{References}

[1] Shannon MA, Bohn PW, Elimelech M et al (2008) Science and technology for water purification in the coming decades. Nature 452 (7185):301-310

[2] Schwarzenbach RP, Escher BI, Fenner K, et al (2006) The challenge of micropollutants in aquatic systems. Science 313 (5790):1072-1077

[3] Elimelech M, Phillip W (2011) The future of seawater desalination: energy, technology, and the environment. Science 333 (6043):712-717

[4] Lewis N.S (2007) Toward cost-effective solar energy use. Science 315 (5813):798-801

[5] Ghasemi H, Ni G, Marconnet A M et al (2014) Solar steam generation by heat localization. Nat commun 5: 4449.

[6] Liu G, Chen T, Xu J, Li G, Wang K (2020) Solar evaporation for simultaneous steam and power generation. J Mater Chem A 8(2): 513-531.

[7] Liu G, Xu J, Wang K (2017) Solar water evaporation by black photothermal sheets. Nano Energy 41: 269-284. 
[8] Wu S, Xiong G, Yang H et al (2019) Multifunctional Solar Waterways: Plasma-Enabled Self-Cleaning Nanoarchitectures for Energy-Efficient Desalination. Adv Energy Mater 1901286

[9] Chen Z, Dang B, Luo X et al (2019) Deep Eutectic Solvent-Assisted In Situ Wood Delignification: A Promising Strategy To Enhance the Efficiency of Wood-Based Solar Steam Generation Devices. ACS Appl Mater Inter 11 (29): 26032-26037

[10]Qiu P, Liu F, Xu C et al (2019) Porous three-dimensional carbon foams with interconnected microchannels for high-efficiency solar-to-vapor conversion and desalination. J Mater Chem A 7 (21): 13036-13042

[11]Chen R, Wang X, Gan Q et al (2019) A bifunctional $\mathrm{MoS}_{2}$-based solar evaporator for both efficient water evaporation and clean freshwater collection. J Mater Chem A 7 (18): $11177-11185$

[12] Yang X, Yang Y, Fu L et al (2018) An Ultrathin Flexible 2D Membrane Based on Single - Walled Nanotube--MoS 2 Hybrid Film for High-Performance Solar Steam Generation. Adv Funct Mater 28 (3): 1704505

[13]Bae K, Kang G, Cho SK et al (2015) Flexible thin-film black gold membranes with ultrabroadband plasmonic nanofocusing for efficient solar vapour generation. Nat Commun 6: 10103

[14]Liu H, Chen C, Wen H et al (2018) Narrow Bandgap Semiconductor Decorated Wood Membrane for High-Efficiency Solar-Assisted Water Purification. J Mater Chem 6 (39):18839-18846 
[15]Li X, Yao Z, Wang J, Li D, Jiang Z (2019) A Novel Flake-like Cu7 $\mathrm{S}_{4}$ Solar Absorber for High-Performance Large-Scale Water Evaporation. Acs Appl Energy Mater 2 (7):51545161

[16]Zhu G, Xu J, Zhao W, Huang F (2016) Constructing Black Titania with Unique Nanocage Structure for Solar Desalination. ACS Appl Mater Inter 8 (46):31716-31721

[17]Tahir Z, Kim S, Ullah F et al (2020) Highly Efficient Solar Steam Generation by Glassy Carbon Foam Coated with Two-Dimensional Metal Chalcogenides. ACS Appl Mater Inter $12(2): 2490-2496$

[18]Liu C, Cai C, Ma F, Zhao X, Ahmad H (2020) Accelerated solar steam generation for efficient ions removal. J Colloid Interf Sci 560:103-110

[19]Wang J, Li Y, Deng L et al (2017) High-Performance Photothermal Conversion of Narrow-Bandgap $\mathrm{Ti}_{2} \mathrm{O}_{3}$ Nanoparticles. Adv Mater 29 (3):1603730

[20]Gao M, Zhu L, Peh CKN, Ho GW (2019) Solar absorber material and system designs for photothermal water vaporization towards clean water and energy production. Energ Environ Sci 12 (3):841-864

[21]Yang HC, Hou J, Chen V, Xu ZK (2016) Janus membranes: exploring duality for advanced separation. Angew Chem Int Edit 55 (43):13398-13407

[22]Zheng Z, Nottbohm CT, Turchanin A et al (2010) Janus nanomembranes: a generic platform for chemistry in two dimensions. Angew Chem Int Ed 49 (45):8493-8497

[23] Cho I, Lee KW (1985) Morphology of latex particles formed by poly (methyl methacrylate)-seeded emulsion polymerization of styrene. J Appl Polym Sci 30 (5):19031926 
[24]Zhang Z, Kong XY, Xiao K et al (2016) A bioinspired multifunctional heterogeneous membrane with ultrahigh ionic rectification and highly efficient selective ionic gating. Adv Mater 28 (1):144-150

[25]Hou J, Ji C, Dong G et al (2015) Biocatalytic Janus membranes for $\mathrm{CO}_{2}$ removal utilizing carbonic anhydrase. J Mater Chem A 3 (33):17032-17041

[26]Cao M, Xiao J, Yu C, Li K, Jiang L (2015) Hydrophobic/hydrophilic cooperative Janus system for enhancement of fog collection. Small 11 (34):4379-4384

[27] Xu W, Hu X, Zhuang S et al (2018) Flexible and salt resistant Janus absorbers by electrospinning for stable and efficient solar desalination. Adv Energy Mater 8 (14): 1702884

[28] Yang Y, Yang X, Fu L et al (2018) Two-Dimensional Flexible Bilayer Janus Membrane for Advanced Photothermal Water Desalination. ACS Energy Lett 3(5):1165-1171

[29]Hu R, Zhang J, Kuang Y et al (2019) A Janus evaporator with low tortuosity for long-term solar desalination. J. Mater Chem A 7 (25):15333-15340

[30]Qin D, Zhu Y, Yang R, Xiong Z (2020). A salt-resistant Janus evaporator assembled from ultralong hydroxyapatite nanowires and nickel oxide for efficient and recyclable solar desalination. Nanoscale Doi:10.1039/c9nr10357k

[31]Wang Y, Zhang L, Wang P (2016) Self-Floating Carbon Nanotube Membrane on Macroporous Silica Substrate for Highly Efficient Solar-Driven Interfacial Water Evaporation. ACS Sustainable Chem Eng 4 (3):1223-1230

[32] Yin Z, Wang H, Jian M et al (2017) Extremely Black Vertically Aligned Carbon Nanotube Arrays for Solar Steam Generation. ACS Appl Mater Inter 9 (34):28596-28603 
[33]Li X, Xu W, Tang M et al (2016) Graphene oxide-based efficient and scalable solar desalination under one sun with a confined 2D water path. Proc Natl Acad Sci U.S.A. 113 (49):13953-13958

[34]Ito Y, Tanabe Y, Han J et al (2015) Multifunctional Porous Graphene for High-Efficiency Steam Generation by Heat Localization. Adv. Mater 27 (29):4302-4307

[35]Chen R, Wu Z, Zhang T, Yu T, Ye M (2017) Magnetically recyclable self-assembled thin films for highly efficient water evaporation by interfacial solar heating. RSC Adv 7 (32):19849-19855

[36]Wang Z, Liu Y, Tao P et al (2014) Bio-Inspired Evaporation Through Plasmonic Film of Nanoparticles at the Air-Water Interface. Small 10 (16):3234-3239

[37]Chen T, Sun H, Mu P et al (2020) Fatty amines as a new family of organic phase change materials with exceptionally high energy density. Sol Energ Mat Sol C 206:110340

[38]Chen L, Xia M, Du J, Luo X, Li A (2019) Superhydrophilic and oleophobic porous architectures based on basalt fibers as novel oil-repellent photothermal materials for efficient solar steam generation. ChemSusChem 13: 493-500

[39] Mu P, Bai W, Fan Y et al (2019) Conductive hollow kapok fiber-PPy monolithic aerogels with excellent mechanical robustness for efficient solar steam generation. J Mater Chem A 7 (16):9673-9679

[40]He J, Zhao G, Mu P et al (2019) Scalable fabrication of monolithic porous foam based on cross-linked aromatic polymers for efficient solar steam generation. Sol Energ Mat Sol C 201:110111

[41] Sharshir SW, Peng G, Wu L et al (2017) The effects of flake graphite nanoparticles, phase change material, and film cooling on the solar still performance. Appl Energ 191:358-366 
[42]Peng G, Deng S, Sharshir SW et al (2020) High efficient solar evaporation by airing multifunctional textile. Int J Heat Mass Tran 147:118866

[43] Sharshir SW, Yang N, Peng G, Kabeel AE (2016) Factors affecting solar stills productivity and improvement techniques: A detailed review. Appl Therm Eng 100:267284

[44]Peng G, Ding H, Sharshir SW et al (2018) Low-cost high-efficiency solar steam generator by combining thin film evaporation and heat localization: Both experimental and theoretical study. Appl Therm Eng 143:1079-1084

[45]Ni GW, Zandavi SH, Javid SM et al (2018) A Salt-Rejecting Floating Solar Still for LowCost Desalination. Energy Environ Sci 11(6): 1510-1519

[46] Kashyap V, Al-Bayati A, Sajadi SM et al (2017) Flexible Anti-Clogging Graphite Film for Scalable Solar Desalination by Heat Localization. J. Mater Chem A 5(29): 15227-15234

[47]Zeng J, Wang Q, Shi Y, Liu P, Chen R (2019) Osmotic Pumping and Salt Rejection by Polyelectrolyte Hydrogel for Continuous Solar Desalination. Adv Energy Mater 9(38): 1900552

[48] Aviles F, Cauichrodriguez JV, Mootah L, Maypat A, Vargascoronado R (2009) Evaluation of mild acid oxidation treatments for MWCNT functionalization. Carbon 47 (13):29702975

[49]Ma S, Yong D, Zhang Y, Wang X, Han X (2014) A Universal Approach for the Reversible Phase Transfer of Hydrophilic Nanoparticles. Chem Eur J 20 (47):15580-15586 [50]Vicentini DS, Barra GM, Bertolino JR, Pires A (2007) Polyaniline/thermoplastic polyurethane blends: Preparation and evaluation of electrical conductivity. Eur Polym J 43 (10):4565-4572 
[51]Ma S, Wang Y, Jiang K, Han X (2015) Decoratable hybrid-film-patch stabilized Pickering emulsions and their catalytic applications. Nano Res 8 (8):2603-2610

[52]Wang G, Fu Y, Guo A et al (2017) Reduced graphene oxide-polyurethane nanocomposite foam as a reusable photoreceiver for efficient solar steam generation. Chem Mater 29 (13):5629-5635.

[53]Zhao Q, Du C, Jia Y et al (2020) Solar-powered Janus membrane for one-step conversion of sewage to clean water. Chemical Engineering Journal 387:124131

[54]Yu H-H, Yan L-J, Shen Y-C, Chen S-Y, Xu Z-K (2020) Janus Poly(Vinylidene Fluoride) Membranes with Penetrative Pores for Photothermal Desalination. Research Doi: $10.34133 / 2020 / 3241758$ 